\title{
Electronic spectra of $\mathrm{Yb}^{2+}$-doped $\mathrm{SrCl}_{2}$.
}

\author{
Goar Sánchez-Sanz, ${ }^{1}$ Luis Seijo, ${ }^{1,2}$ and Zoila Barandiarán ${ }^{1,2, *}$ \\ ${ }^{1}$ Departamento de Química, Universidad Autónoma de Madrid, 28049 Madrid, Spain \\ ${ }^{2}$ Instituto Universitario de Ciencia de Materiales Nicolás Cabrera, \\ Universidad Autónoma de Madrid, 28049 Madrid, Spain
}

(Dated: August 26, 2010)

\begin{abstract}
The absorption and emission spectra of $\mathrm{Yb}^{2+}$-doped $\mathrm{SrCl}_{2}$ have been calculated on the basis of $a b$ initio quantum chemical calculations which consider recently found, unexpected excited states with double-well energy curves and complex electronic structure, resulting from avoided crossings between Yb-trapped excitons and Yb impurity states, which influence prominent spectral features. The root mean square deviation and largest absolute error of the calculated energy levels are 394 and $-826 \mathrm{~cm}^{-1}$, respectively. The $\mathrm{YbCl}_{8}$ moiety breathing mode vibrational frequencies and bond lengths of the lowest states are consistent with observed vibrational progressions and energy shifts induced by uniaxial compression. Photoionization is predicted above $49000 \mathrm{~cm}^{-1}$ as a consequence of the spin-orbit induced spreading of the Yb-trapped exciton character in the upper part of the spectrum and three new emission bands are predicted with origins at about 33800, 36400, and $43600 \mathrm{~cm}^{-1}$. The electron correlation methods used overestimate the relative stabilization of the $4 f^{14}$ ground state and this leads to a constant error of the whole absorption spectrum of about $3500 \mathrm{~cm}^{-1}(23-7 \%)$. Although this energy shift is customarily considered an adjustable parameter, it is a non-parametric, direct product in an ab initio route which shows the limitations on the proper representation of differential correlation between the $4 f^{N}$ and $4 f^{N-1} 5 d$ (or similar) configurations and the need for theoretical improvement.
\end{abstract}




\section{INTRODUCTION}

Many proven and potential technological applications of lanthanide ions in solids are linked to their numerous electronic excited states. ${ }^{1-3}$ Mastering their optical properties is the goal leading a large amount of basic and applied experimental research, and electronic spectroscopies are the classical and most widely used research tools. First principles electronic structure methods can also contribute to the leading goal because they can be applied to these systems to calculate the electronic structure of their excited states and facilitate analyses, interpretations, and predictions of their spectral features, which may complement and enrich the experimental information and knowledge. This complementarity is most necessary when the spectral features become more complex and the empirical models for interpretation fail because the electronic structure of the excited manifolds of the doped lanthanide ion departs strongly from their atomic counterparts and becomes very ligand and host dependent (such as is the case of the excited $4 f^{N-1} 5 d$ manifolds), a characteristic which, however, makes them very attractive on the line of tuning the optical properties.

All the excited states of $\mathrm{Yb}^{2+}$-doped crystals belong to this category because the only member of the atomic-like $4 f^{N}$ manifold is the $4 f^{14}$ ground state. ${ }^{4-11}$ As a consequence, notable host variations of its spectral features have been reported and reviewed. ${ }^{10,12}$ Furthermore, a recent $a b$ initio quantum chemical study of the excited states of $\mathrm{Yb}^{2+}$-doped $\mathrm{SrCl}_{2}$ crystals conducted in our laboratory (Ref. 13; Paper I, from now on) has revealed the existence of unexpected excited states, whose electronic structure departs drastically from any of the free ion. The new excited states show double-well potential energy surfaces and dual electronic structure resulting from avoided crossings between Yb-trapped exciton states and $4 f^{13} 6 s$ states. The former are pre-ionization states; the latter differ markedly in energy from their free ion parents. The double-well excited states lie above and close in energy to the $4 f^{13} 5 d$ manifold, with which they interact strongly through spin-orbit coupling. Altogether, the resulting excited state electronic structure of $\mathrm{Yb}^{2+}$-doped $\mathrm{SrCl}_{2}$ crystals is far more complex than deduced from experiments and their empirical analyses. ${ }^{5,7,10}$ These findings urge the calculation and reinterpretation of the absorption and emission spectra of $\mathrm{Yb}^{2+}$-doped $\mathrm{SrCl}_{2}$ crystals within the new excited state scenario, and these are the main objectives of this paper.

A summary of the electronic structure properties of all the excited states found in $\mathrm{Yb}^{2+}$. 
doped $\mathrm{SrCl}_{2}$ in Paper I is presented in Sec. II A. The results of the calculation of the absorption spectrum are confronted with the available experimental data and interpretations in Sec. II B, where the impact of the new unexpected electronic structures on the spectral features is discussed in detail. This results in different assignments to the ones accepted so far and prediction of photoionization above $49000 \mathrm{~cm}^{-1}$. The calculated and experimental emission spectra are discussed together in Sec. II C. The calculations predict the existence of new emission bands which should be detected and studied experimentally.

\section{RESULTS AND DISCUSSION}

\section{A. Electronic structure of the excited states of $\mathrm{Yb}^{2+}$-doped $\mathrm{SrCl}_{2}$.}

The spin-orbit, correlated $\left(\mathrm{YbCl}_{8} \mathrm{Sr}_{12}\right)^{18+}$ embedded-cluster wavefunctions and potential energy surfaces of the excited states of $\mathrm{Yb}^{2+}$-doped $\mathrm{SrCl}_{2}$ crystals were calculated up to $65000 \mathrm{~cm}^{-1}$ in Paper I. The results of the calculations and their analyses showed a very complex electronic structure due to extensive spin-orbit induced configurational mixings. Without spin-orbit the excited states of $\mathrm{Yb}^{2+}$-doped $\mathrm{SrCl}_{2}$ were found to be grouped in three manifolds, which can be observed on the left of Fig. 1. The first two, in increasing energy order, correspond to states with main configurational character $4 f^{13} 5 d\left(e_{g}\right)$ and $4 f^{13} 5 d\left(t_{2 g}\right)$, respectively. The third one contains excited states with double-well potential energy curves and dual electronic structure, which result from avoided crossings, at a critical $\mathrm{Yb}-\mathrm{Cl}$ distance of $2.89 \AA$, between $4 f^{13} a_{1 g}^{Y b T E}$ Yb-trapped exciton states (more stable at short $\mathrm{Yb}-\mathrm{Cl}$ distances) and $4 f^{13} 6 s$ states (more stable at long $\mathrm{Yb}-\mathrm{Cl}$ distances). The analysis of the electronic density of the impurity trapped excitons showed their pre-ionization character since the excited electron appears to be significantly localized outsite the $\mathrm{YbCl}_{8}$ moiety, on the six next nearest empty interstitial sites of the fluorite-type structure, and their $\mathrm{Yb}-\mathrm{Cl}$ bond lengths (short distance minimum) are very close to that of the $\mathrm{Yb}^{3+}$ doped $\mathrm{SrCl}_{2}$ ground configuration. Four new groups of states are formed as a result of spin-orbit coupling, which affects more strongly the $4 f^{13}$ subshell, splitting it into effective $4 f^{13}(J=7 / 2)$ and $4 f^{13}(J=5 / 2)$ components (right of Fig. 1). Only the first and fourth, in increasing energy order, keep their single configurational character: $4 f^{13}(7 / 2) 5 d\left(e_{g}\right)$ (low-

est energy curves in red on the right of Fig. 1) and $\left[4 f^{13}(5 / 2) a_{1 g}^{Y b T E}-4 f^{13}(5 / 2) 6 s\right]$ (high- 
est double-well energy curves in green on the right of Fig. 1), whereas the second and third show extensive configurational mixing resulting from the interactions between the $4 f^{13}(5 / 2) 5 d\left(e_{g}\right)$ and $4 f^{13}(7 / 2) 5 d\left(t_{2 g}\right)$ submanifolds, and of the $4 f^{13}(5 / 2) 5 d\left(t_{2 g}\right)$ and the double-well $\left[4 f^{13}(7 / 2) a_{1 g}^{Y b T E}-4 f^{13}(7 / 2) 6 s\right]$ submanifolds, respectively.

The existence of the double-well $\left[4 f^{13} a_{1 g}^{Y b T E}-4 f^{13} 6 s\right]$ excited states above and close to the $4 f^{13} 5 d\left(t_{2 g}\right)$ manifold was not expected and was not known before the ab initio calculations of Paper I. However, their peculiar energy curves and dual electronic structure strongly affect the $4 f^{13} 5 d\left(t_{2 g}\right)$ states because they all mix through spin-orbit coupling. Hence, the interpretation of the spectroscopy of the $\mathrm{Yb}^{2+}$-doped $\mathrm{SrCl}_{2}$ material must consider their presence among the excited states of the material. Therefore, the goal of this paper is to calculate, reassign, and reinterpret the absorption and emission spectra of $\mathrm{Yb}^{2+}$-doped $\mathrm{SrCl}_{2}$ using the energies and wavefunctions obtained in Paper I as basic ingredients.

The methods used to calculate the wavefunctions and energies have been described and referred in Paper I. The methods used here to calculate spectral features (band shapes, electric dipole transition moments, oscillator strengths, radiative emission lifetimes) have been described in Refs. 11 and 14. The program EPCISO ${ }^{15}$ has been used to calculate electric dipole transition moments and absorption and emission oscillator strengths.

\section{B. Absorption spectrum of $\mathrm{Yb}^{2+}$-doped $\mathrm{SrCl}_{2}$.}

In this Section we present the results of the calculation of the absorption spectrum of $\mathrm{Yb}^{2+}$-doped $\mathrm{SrCl}_{2}$. First of all we try to assess the accuracy of the calculated energies and structures. Secondly, we describe the three regions in which the absorption spectrum is devided. In each case, we assign the experimental features trying to match theoretical and experimental energies, energy separations, and intensities, we reinterpret the spectrum, and make some predictions that could orient further experimental work. And, finally, we comment on the basic characteristics of excited state absorptions.

\section{Lowest energy levels. Accuracy of the calculations.}

The analysis of the accuracy of the calculations by direct comparisons with available experimental data can be done in two steps which reveal the response of the methods used to 
different demands posed by the non-empirical calculation of the transition energies of $\mathrm{Yb}^{2+}$ doped $\mathrm{SrCl}_{2}$. In a first step, only the more thouroughly studied and precise experimental data are used to compare with the calculated values; this includes some zero-phonon lines and their vibrational sequences. The analyses suggest that there is a systematic, common error in all the transition energies from the ground state, which is interesting to isolate because a number of properties, discussions, and concusions are independent of it. In a second step, the experimental and theoretical energies are referred to the first electric dipole allowed energy level $\left(1 T_{1 \mathrm{u}}\right)$ and, then, they are compared; in this way excited state specific discrepancies are made clear. The first type of analysis is presented in this Section. The second type is presented in the next Section together with the assignment and interpretation of the experimental data.

According to the analyses presented in Paper I, the lowest 13 excited states of $\mathrm{Yb}^{2+}$ doped $\mathrm{SrCl}_{2}$ have a dominant $4 f^{13}(7 / 2) 5 d\left(e_{g}\right)$ configurational character. We present their bond lengths, minimum-to-minimum energy differences relative to the ground state, and the $\mathrm{YbCl}_{8}$ moiety $a_{1 g}$ breathing mode vibrational frequencies in Table I together with available experimental data in order to deduce their accuracy.

Only two electronic origins of $T_{1 \mathrm{u}}$ symmetry have been measured directly from vibrational progressions observed in absorption ${ }^{5,10}$ and emission spectra ${ }^{8,9}$ at low temperatures (Table I); they are assigned to levels $1 T_{1 \mathrm{u}}$ and $4 T_{1 \mathrm{u}}$ in this work (see below) and their calculated energies are overestimated by $3500-3700 \mathrm{~cm}^{-1}$ (see Table I). Analogous error $\left(3161 \mathrm{~cm}^{-1}\right)$ is found for $3 T_{1 \mathrm{u}}$ whose electronic origin has been deduced from the observation of a very weak and broad structure at $28590 \mathrm{~cm}^{-1}$, which was tentatively assigned to the first member of a $213 \pm 3 \mathrm{~cm}^{-1}$ progression. ${ }^{10}$ Two additional origins have been reported $\left(1 E_{\mathrm{u}}\right.$ and $\left.2 T_{2 \mathrm{u}}\right)$, whose zero-phonon lines cannot be observed directly given that the only electric dipole allowed transitions from or to the $A_{1 \mathrm{~g}}$ ground state involve $T_{1 \mathrm{u}}$ states. The energy of the $1 E_{\mathrm{u}}$ zero-phonon line is not known, but it should lie between the highest vibronic origin observed in emission $\left(24958 \mathrm{~cm}^{-1}\right)$ and the lowest vibronic origin observed in absorption $\left(25088 \mathrm{~cm}^{-1}\right)$ at $4.2 \mathrm{~K}$ in Ref. 6 . Its energy has also been estimated from extrapolation of the energy difference between $1 E_{\mathrm{u}}$ and $1 T_{1 \mathrm{u}}$ band maxima from room temperature to 10 K. ${ }^{10}$ The energy of the $2 T_{2 \mathrm{u}}$ origin has been inferred from very low temperature (4.2 and $1.8 \mathrm{~K}$ ) experimental studies of the effects of uniaxial compression and electric and magnetic fields, which have allowed to observe directly its lower symmetry components and their 
vibrational progressions. ${ }^{8}{ }^{8}$ The error of the theoretical values is lower for $1 E_{\mathrm{u}}\left(2800 \mathrm{~cm}^{-1}\right)$ and very similar to those commented above for $2 T_{2 \mathrm{u}}\left(3300 \mathrm{~cm}^{-1}\right)$, and the average error of the calculations for all the origins discussed so far is $3300 \mathrm{~cm}^{-1}$, which corresponds to an overestimation of $10-13 \%$. We have shown in Paper I that the interconfigurational transitions of $\mathrm{SrCl}_{2}: \mathrm{Yb}^{2+}$ dominated by the excitation of an electron from the $4 f$ to the $5 d$ shells increase by $30000 \mathrm{~cm}^{-1}$ with dynamic correlation. This very large effect is associated with the facts that electron correlation is larger for tight $4 f-4 f$ electron pairs than for more distant $4 f-5 d$ pairs and that the number of $4 f-4 f$ pairs substituted is largest in this $14 f$-electron system which is, in this respect, the worst working scenario for electron correlation methods in the $f$-element series. This largely explains the larger than usual 10-13\% discrepancy between theory and experiments.

The energy spacings deduced from the vibrational progressions observed in the experimental spectra we have just referred to, have allowed to determine the totally symmetric vibrational frequency of the $\mathrm{YbCl}_{8}$ moiety in the ground state $1 A_{1 \mathrm{~g}}$ and in three excited states: $1 T_{1 \mathrm{u}}, 3 T_{1 \mathrm{u}}$, and $4 T_{1 \mathrm{u}}$. Comparison of the calculated values with the experimental data show discrepancies of $8-10 \%$ and $3-6 \%$ in the ground and excited states, respectively. The larger disagreement in the ground state could be also related with the limitations in the differential dynamic correlation.

Direct comparisons of the bond lengths with experiments are not possible since extended X-ray absorption fine structure (EXAFS) experiments are not available neither for the ground state nor for the excited states, to our knowledge. However, indirect assessments of their quality are possible. In effect, on the one hand, theoretical studies have pointed out that the sign of pressure-induced $f-d$ transition energy shifts and the sign of the bond length change upon $f-d$ excitation are the same, so that pressure-induced red shifts have been predicted for the lowest $f-d$ transitions of $\mathrm{Ce}^{3+}$-doped $\mathrm{Cs}_{2} \mathrm{NaYCl}_{6}$, where the bond length is shorter in the $d$ state. $^{16}$ These predictions have been confirmed experimentally for $\mathrm{Ce}^{3+}$-doped $\mathrm{Cs}_{2} \mathrm{NaLuCl}_{6}{ }^{17}$ and the same relationship has been found in cubic coordination for $\mathrm{Sm}^{2+}$-doped $\mathrm{SrF}_{2} \cdot{ }^{18,19}$ In this line, a strong red shift $\left(11 \mathrm{~cm}^{-1}\right)$ and a slight splitting $\left(\sim 1.5 \mathrm{~cm}^{-1}\right)$ of the $1 A_{1 \mathrm{~g}}-1 T_{1 \mathrm{u}}$ zero-phonon absorption band at $27045 \mathrm{~cm}^{-1}$ has been reported for $\mathrm{SrCl}_{2}: \mathrm{Yb}^{2+}$ in Ref 8 , as a result of uniaxial pressure $\left(\mathrm{P}_{\langle 100\rangle}=10 \mathrm{~kg} / \mathrm{mm}^{2}\right)$ at $4.2 \mathrm{~K}$. This observed red shift supports the negative sign of the bond length change upon $1 A_{1 \mathrm{~g}}-1 T_{1 \mathrm{u}}$ excitation that can be deduced from the bond lengths presented in Table I: $-0.055 \AA$. On the 
other hand, the magnitude (the square) of the bond length change sustantially controlls the relative intensities in vibrational sequences constructed upon an electronic origin; therefore, it is useful to compare the experimental ${ }^{9}$ and theoretical band shapes associated with the first $T_{1 \mathrm{u}}$ level in Fig. 2. A good agreement is observed as the height of the $0-0,0-1$, and $0-2$ members decreases in both spectra, and the sequence intervals are very close, as commented above, which suggests that the absolute value of the bond distance offset $\left|\Delta R_{e}\left(1 T_{1 \mathrm{u}}\right)\right|=$ $\left|R_{e}\left(1 T_{1 \mathrm{u}}\right)-R_{e}\left(1 A_{1 \mathrm{~g}}\right)\right|=0.055 \AA$, is also quite good. However, it should be noted that the origin line appears to be narrower than the others in the experimental band, a fact that was stressed in Refs. 5 and 6, and that the experimental envelope includes other sequences as well. This suggests that the relative intensity of the 0-0 line could be lower than suggested by its relative height, which would indicate that a larger distance offset (in absolute value), such as that used to produce the band envelope in the inset of Fig. $2\left|\Delta R_{e}\left(1 T_{1 \mathrm{u}}\right)\right|=0.075 \AA$, might be more accurate. Therefore, we should conclude that the error of the theoretical value is of the order of $0.02 \AA$. This should also apply to the bond distance offsets of the other $4 f^{13}(7 / 2) 5 d\left(e_{g}\right)$ electronic states presented in Table I.

\section{Ground state absorption spectrum.}

We present the calculated energy levels corresponding to the electric dipole allowed transitions $1 A_{1 \mathrm{~g}} \rightarrow \mathrm{i} T_{1 \mathrm{u}}$ in Table II together with the experimental values reported in Ref. 5 (also observed in Refs. 10 and 7) and some additional data from Refs. 10 and 7. Their energies are referred to the $1 T_{1 \mathrm{u}}$ zero-phonon line to make the direct comparison with experiments easier. This is convenient taking into account the systematic overestimation of the calculated zero-phonon lines discussed above. The experimental energies correspond to band maxima and are referred to the experimental $1 T_{1 \mathrm{u}}$ zero-phonon line observed at $27049 \mathrm{~cm}^{-1}$, from Ref. 5, unless otherwise specified. The relative oscillator strengths, $f_{i} / f_{1}$, are also given in Table II. Their experimental values from Ref. 5 were obtained after background corrections which could not be determined with great precision and, hence, are not known with great accuracy. ${ }^{5}$ Different background corrections were applied to the experimental values of Ref. 5 in Ref. 10 and the corresponding $f_{i} / f_{1}$ data are also given in Table II when different from those of Ref. 5. $\mathrm{Yb}-\mathrm{Cl}$ equilibrium distance offsets with respect to the ground state are also given in Table II, where $\Delta R_{e}\left(\mathrm{i} T_{1 \mathrm{u}}\right)=R_{e}\left(\mathrm{i} T_{1 \mathrm{u}}\right)-R_{e}\left(1 A_{1 \mathrm{~g}}\right)$. Their square values determine 
the structure of the $\mathrm{YbCl}_{8}$ moiety $a_{1 g}$ vibrational progressions as commented above. The calculated absorption spectrum envelope is presented in Fig. 3; it was produced as a superposition of the $a_{1 g}$ vibrational progression of each individual $\mathrm{i} T_{1 \mathrm{u}}$ origin using the spectroscopic constants tabulated in Table III of Paper I and Table II of this work, and the semiclassical time-dependent approach of Heller with different values for the line broadening parameter (35 and $5 \mathrm{~cm}^{-1}$ in the low- and high-resolution spectra, respectively). ${ }^{20-22}$ The lower energy scale is referred to the $1 T_{1 \mathrm{u}}$ zero-phonon line; the upper one shows the calculated energies shifted by $-3500 \mathrm{~cm}^{-1}$. The energy curves of all $\mathrm{i} T_{1 \mathrm{u}}$ states have been plotted in the inset to facilitate discussions; more detailed figures including all spin-orbit states and analyses of their interactions and electronic structure are given in Paper I.

The calculated absorption spectrum is divided in three regions. In the first one, Region 1, only one band is observed which results from the superposition of the first four $T_{1 \mathrm{u}}$ origins, and is dominated by the most intense $1 T_{1 \mathrm{u}}$ progression (see Fig. 3 and Table II). Only a few electronic states of $\mathrm{SrCl}_{2}: \mathrm{Yb}^{2+}$ have a dominant configurational character (cf. Paper I) and this is the case of the first four $T_{1 \mathrm{u}}$ excited states, which are mainly $4 f^{13}(7 / 2) 5 d\left(e_{g}\right)$. The first origin, $1 T_{1 \mathrm{u}}$, has been discussed above and the second, $2 T_{1 \mathrm{u}}$, has not been detected experimentally probably because it lies about $670 \mathrm{~cm}^{-1}$ above $1 T_{1 \mathrm{u}}$ and, hence, is immersed in the $a_{1 g}$ and other vibrational progressions of $1 T_{1 \mathrm{u}}$ (see Fig. 2). ${ }^{5,9,10}$ The bands associated with $3 T_{1 \mathrm{u}}$ and $4 T_{1 \mathrm{u}}$ have been detected experimentally and can be easily assigned by our calculations. The $2010 \mathrm{~cm}^{-1}$ value of Ref. 5 given in Table II corresponds to the difference of $4 T_{1 \mathrm{u}}$ and $1 T_{1 \mathrm{u}}$ band maxima; this difference should be very close to their zero-ponon line difference given that both states have very similar vibrational progressions, ${ }^{5,10}$ in good correspondence with their analogous equilibrium distance offset with respect to the ground state (-0.059 and $-0.055 \AA$, respectively). The assignment we have just discussed leads to the following maximum and mean absolute error in the energies: 320 and $254 \mathrm{~cm}^{-1}$, respectively.

The second region of the absorption spectrum, Region 2, starts some $5000 \mathrm{~cm}^{-1}$ above, extends from 7500 to $14500 \mathrm{~cm}^{-1}$ above $1 T_{1 \mathrm{u}}$, and comprises the $\mathrm{i} T_{1 \mathrm{u}}$ ( $\mathrm{i}=5$ to 13 ) origins grouped in four bands formed by the superposition of origins $5 ; 7$ and 8;10,11, and 12; and 13, all of them with non-negligible intensity, whose vibrational progressions are dominated by the zero-phonon line, and of which the $13 T_{1 u}$ band is much more intense than the rest. Analogous energy range, bands separations, and overall intensity pattern apply to the experimental data reported in Ref. 5 and 10, so that their assignment based on the calculated 
energies and relative oscillator strengths is not difficult and lead to the following maximum and mean absolute error for their energies: 484 and $310 \mathrm{~cm}^{-1}$, respectively (see Table II). Two additional, very weak peaks have been reported by $\operatorname{Loh}^{7}$ which lie in the large energy gap between $13 T_{1 \mathrm{u}}$ and $14 T_{1 \mathrm{u}}$. These signals have not been reported in Refs. 5 and 10 and do not match any of the calculated values.

The $\mathrm{i} T_{1 \mathrm{u}}(\mathrm{i}=5$ to 13$)$ excited states responsible for Region 2 result from spin-orbit induced $4 f^{13}(5 / 2) 5 d\left(e_{g}\right)$ and $4 f^{13}(7 / 2) 5 d\left(t_{2 g}\right)$ configurational mixing (cf. Paper I) and their $\mathrm{Yb}-\mathrm{Cl}$ bond lengths take intermediate values with respect to the spin-orbit free $4 f^{13} 5 d\left(e_{g}\right)$ and $4 f^{13} 5 d\left(t_{2 g}\right)$ states (see inset of Fig. 3). As a consequence, the equilibrium distance offsets are found to lie close to the middle of the spin-orbit free average values $\Delta R_{e}\left[4 f^{13} 5 d\left(e_{g}\right)\right]$ $=-0.055$ and $\Delta R_{e}\left[4 f^{13} 5 d\left(t_{2 g}\right)\right]=+0.013 \AA$ (cf. Paper I), and are noticeably smaller than the offsets of states $1 T_{1 \mathrm{u}}$ to $4 T_{1 \mathrm{u}}$. As a result, the 0-0 line becomes the most intense and only member of the $a_{1 \mathrm{~g}}$ vibrational progressions of $1 A_{1 \mathrm{~g}}-\mathrm{i} T_{1 \mathrm{u}}$ ( $\mathrm{i}=5$ to 13 ) transitions, as commented above, in contrast with the progressions observed (both theoretically and experimentally) for the lower $1-4 T_{1 \mathrm{u}}$ states (cf. spectra of Figs. 2 and 3). This explains why vibrational progressions have not been observed in this part of the absorption spectrum whereas they have been detected on the $1 T_{1 \mathrm{u}}$ and $4 T_{1 \mathrm{u}}$ lower origins. ${ }^{5,7,10}$ This also means that the peak energies given in Table 1 of Ref. 5 should correspond to zero-phonon lines, according to the calculations, so that the energy differences given in Table II for the $\mathrm{i} T_{1 \mathrm{u}}$ ( $\mathrm{i}=5$ to 13 ) levels correspond to zero-phonon line differences and are directly comparable with the theoretical values. The relatively large intensity of the $13 T_{1 \mathrm{u}}$ origin (calculated and measured) is due to the significant contribution (13\%) of the enabling term $5^{1} T_{1 \mathrm{u}}$ to its spin-orbit wave function. In effect, on the one hand ${ }^{1} P_{u}$ is the only $4 f^{13} 5 d$ state which leads to electric dipole allowed transition from the $4 f^{14}{ }^{1} S_{g}$ ground state in the $\mathrm{Yb}^{2+}$ free ion without spin-orbit coupling. On the other hand, $5^{1} T_{1 \mathrm{u}}$ is the $4 f^{13} 5 d^{1} T_{1 \mathrm{u}}$ state of the $\mathrm{Yb}^{2+}$ doped crystal which should keep the largest atomic $4 f^{13} 5 d-{ }^{1} P_{u}$ character, as discussed in Ref. 23. Therefore, $5^{1} T_{1 \mathrm{u}}$ is the most efficient electric dipole transition enabling ${ }^{1} T_{1 \mathrm{u}}$ term as it transports the favorable ${ }^{1} S_{g}-{ }^{1} P_{u}$ atomic selection rule onto the crystal transitions. This same argument explains the high intensity of $14 T_{1 \mathrm{u}}\left(26 \% 5^{1} T_{1 \mathrm{u}}\right)$ and $17 T_{1 \mathrm{u}}\left(44 \% 5^{1} T_{1 \mathrm{u}}\right)$, as we comment below.

The third and highest region in the theoretical spectrum, Region 3, starts some $2500 \mathrm{~cm}^{-1}$ above the end of Region 2, between 17700 and $23500 \mathrm{~cm}^{-1}$ above $1 T_{1 \mathrm{u}}$ (see Fig. 3 and 
Table II) and comprises the $\mathrm{i} T_{1 \mathrm{u}}(\mathrm{i}=14$ to 20$)$ origins grouped in four bands formed by the superposition of origins 14; 16; 17; and 19 and 20, all of them with non-negligible intensity. Their vibrational progressions are very different, the $14 T_{1 \mathrm{u}}$ and $17 T_{1 \mathrm{u}}$ bands are much more intense than the rest, and the latter is the most intense in the whole absorption spectrum. Again, the matching with experimental band energies, separations, and relative oscillator strengths allow to assign the experimental data reported in Ref. 5 and 10 without difficulty, as we comment below (see Table II), which leads to the following maximum and mean absolute error for their energies: 826 and $427 \mathrm{~cm}^{-1}$, respectively. Two additional, very weak peaks have been reported by $\operatorname{Loh}^{7}$ which lie in the neighborhood of bands $17 T_{1 u}$ and $19 T_{1 \mathrm{u}}$.

The $\mathrm{i} T_{1 \mathrm{u}}$ ( $\mathrm{i}=14$ to 20 ) states result from spin-orbit coupling induced configurational mixing between double-well $\left[4 f^{13}(7 / 2) a_{1 g}^{Y b T E}-4 f^{13}(7 / 2) 6 s\right]$ and $4 f^{13}(5 / 2) 5 d\left(t_{2 g}\right)$ states, which makes their potential energy surfaces very anharmonic (see inset in Fig. 3). The bond length offsets included in Table II, which correspond to their long distance minima, vary widely between the values of the offset of the parent double-well $\left[4 f^{13} a_{1 g}^{Y b T E}-4 f^{13} 6 s\right]$ and $4 f^{13} 5 d\left(t_{2 g}\right)$ potential energy surfaces: $\Delta R_{e}\left[4 f^{13} 6 s\right]=+0.122 \AA$ (long distance minimum) and $\Delta R_{e}\left[4 f^{13} 5 d\left(t_{2 g}\right)\right]=+0.013 \AA$, respectively (see Table II). Also, the composition of the spin-orbit wavefunctions vary sharply with the $\mathrm{Yb}-\mathrm{Cl}$ distance, following the irregular shape of the energy curves, as it can be seen in Table III. All these characteristics affect the absorption bands as commented next. It should be noted that the anharmonicities of the energy curves and the strong variation of the character of the spin-orbit wave functions of the excited states with the $\mathrm{Yb}-\mathrm{Cl}$ distance, which makes the electric dipole transition moment $\mathrm{Yb}-\mathrm{Cl}$ distance dependent, make the method of calculation of the band profiles used here $e^{20-22}$ more approximate in this part of the spectrum. Nevertheless, the following discussion and conclusions should be valid; an indication of this is that the discrepancies with experiment do not change drastically.

The $14 T_{1 \mathrm{u}}$ band shape is determined by a large absorption oscillator strength and a large value of its bond length offset relative to the ground state (Table II). The former is calculated at the $\mathrm{Yb}-\mathrm{Cl}$ equilibrium distance of the ground state, at which, the contribution of the $4 f^{13} 5 d\left(t_{2 g}\right) 5^{1} T_{1 \mathrm{u}}$ enabling term to the spin-orbit wave function of main character $4 f^{13} 5 d\left(t_{2 g}\right)$ is substantial: $26 \% 5^{1} T_{1 \mathrm{u}}$ (Table III); the latter is due to the dominance of the $4 f^{13} 6 s$ character in the wave function at longer distances (see inset of Fig. 3 and Table III). 
As a consequence of the latter, the $a_{1 g}$ vibrational sequence is very extended including up to the 0-7 member and having the peak intensity on the $0-3$ member, about $18400 \mathrm{~cm}^{-1}$ above the $1 T_{1 \mathrm{u}}$ zero-phonon line, $362 \mathrm{~cm}^{-1}$ below the experimental peak position (note that for this reason we calculate the theoretical error using the peak energy $18400 \mathrm{~cm}^{-1}$ instead of the zero-phonon energy $17711 \mathrm{~cm}^{-1}$ in Table II).

The bond length offset values decrease for the remaining bands (16, 17, and 20 and $21 T_{1 \mathrm{u}}$ ). The one of $16 T_{1 \mathrm{u}}$ is analogous to those of the lowest $1-4 T_{1 \mathrm{u}}$ levels, so that, following the discussion above, the theoretical values are compared with the experimental energy difference between $16 T_{1 \mathrm{u}}$ and $1 T_{1 \mathrm{u}}$ band maxima in Table II. The remaining bands (17 to 20) are dominated by their zero-phonon line and their equilibrium distance offset suggests that the experimental peaks correspond to electronic origins. The intensity of $17 T_{1 \mathrm{u}}$ stems from the $44 \% 5^{1} T_{1 \mathrm{u}}$ contribution of its wave function at the distance where the absorption oscillator strength is calculated.

A notable feature of the theoretical and experimental spectra ${ }^{5,10}$ is that the intensity is largest at the end of the spectrum. This result is much more evident in the theoretical calculation, which does not include the contribution of other vibrational sequences nor background effects on the calculation of the relative intensities. In effect, the calculated oscillator strengths of the most intense bands $\left(13,14\right.$, and $\left.17 T_{1 \mathrm{u}}\right)$ are around one order of magnitude larger than the rest. This result contrasts with the calculated relative oscillator strengths of the absorption spectrum of $\mathrm{Yb}^{2+}$-doped $\mathrm{CsCaBr}_{3}$ cubic crystal, in which $\mathrm{Yb}^{2+}$ occupies $\mathrm{Ca}$ sites of $O_{h}$ symmetry in hexabromide coordination. ${ }^{23} \mathrm{In}_{\mathrm{CsCaBr}}$, the intensity is also larger at the end of the spectrum, but only by a factor of 2 to $3{ }^{23}$ The results of the calculations in the two systems show a much smaller coupling and energy spread of the five ${ }^{1} T_{1 \mathrm{u}}$ crystal components of the $4 f^{13} 5 d{ }^{1} H,{ }^{1} G,{ }^{1} F$, and ${ }^{1} P$ atomic terms of $\mathrm{Yb}^{2+}$ in the $\mathrm{SrCl}_{2}$ crystal than in the $\mathrm{CsCaBr}_{3}$ crystal $\left[T_{e}\left(5^{1} T_{1 \mathrm{u}}\right)-T_{e}\left(1 T_{1 \mathrm{u}}\right)\right.$ is 16300 and $24600 \mathrm{~cm}^{-1}$, respectively]. As a consequence, whereas the enabling character of the highest atomic ${ }^{1} P$ term is effectively retained in the highest $5^{1} T_{1 \mathrm{u}}$ term of $\mathrm{SrCl}_{2}$, so that a marked dependence of the intensity on this term's contribution to the spin-orbit wave function is observed, it is much more spread to the lower ${ }^{1} T_{1 \mathrm{u}}$ states in $\mathrm{Yb}^{2+}$-doped $\mathrm{CsCaBr}_{3}$ than in $\mathrm{Yb}^{2+}$-doped $\mathrm{SrCl}_{2}$, which enhances their electric dipole transition enabling character. In any case, it should be stressed that the maximum ${ }^{1} T_{1 \mathrm{u}}$ contribution to all the $T_{1 \mathrm{u}}$ spin-orbit states of $\mathrm{Yb}^{2+}$-doped $\mathrm{CsCaBr}_{3}$ and $\mathrm{SrCl}_{2}$ is never high $(<44 \%)$, which points out the inaccuracy of the generalized idea that 
the strong absorption bands of heavy lanthanides $(\mathrm{N}>7)$ are due to excitations to low-spin excited states and are, therefore, spin-allowed transitions. As shown in Ref. 11 and here, they all get their intensity from rather small contribution of spin-enabling terms and therefore, they are spin-enabled transitions, and the enabling character is more or less spread over the ${ }^{1} T_{1 \mathrm{u}}$ terms depending on their coupling in the crystal host.

It is also important to stress the Yb-trapped exciton character of the states responsible of the high energy end of the spectrum. This character appears at short distances and energies higher than $22000 \mathrm{~cm}^{-1}$ above $1 T_{1 \mathrm{u}}$, about $49000 \mathrm{~cm}^{-1}$ above the ground state. The Ybtrapped exciton electronic structures correspond to preionization states, as shown in Paper I. Therefore, their presence suggests that there is a significant probability of photoionization when the sample is irradiated to produce the high energy end of the absorption spectrum. The present calculations do not allow to quantify the extent of such photoionization process.

Finally, we should contrast our assignment of the absorption bands with the assignments of Refs. 5, 10, and 7. Piper et al. ${ }^{5}$ explained all the signals observed as due to excitations to states of the $4 f^{13} 5 d$ configurations only. Pan et $a l .{ }^{10}$ reinterpreted the spectrum assigning the peaks at 7989 and $18745 \mathrm{~cm}^{-1}$ above $1 T_{1 \mathrm{u}}$ to excitations to $4 f^{13}(7 / 2) 6 s$ and $4 f^{13}(5 / 2) 6 s$ levels, respectively, because their energies match their corresponding values in the free $\mathrm{Yb}^{2+}$ ion. Our results agree with this reinterpretation only partially: On the one hand, we also find the $4 f^{13} 6 s$ character in some excited states, but, on the other hand, the $4 f^{13}(7 / 2) 6 s$ character is not found below the crystal level $14 T_{1 \mathrm{u}}$, as discussed above. In particular, our calculations do not support the assignment of the feature at $7989 \mathrm{~cm}^{-1}$ above $1 T_{1 \mathrm{u}}$ to $4 f^{13}(7 / 2) 6 s$ and support the assignment of the $18745 \mathrm{~cm}^{-1}$ feature to the $4 f^{13} 6 s$ configuration, but we assign it to one of the lower spin-orbit components, $4 f^{13}(7 / 2) 6 s$, instead of to one of the higher $4 f^{13}(5 / 2) 6 s$. The interactions which destabilize so strongly the states of the $4 f^{13} 6 s$ configuration from the free ion to the embedding host have been discussed in detail elsewhere. ${ }^{24}$ The disagreements between our assignments and those by $\operatorname{Loh}^{7}$ are more extensive. Our calculations rule out the need to invoke the existence of three different $\mathrm{Yb}^{2+}$ sites plus three different $\mathrm{Yb}^{2+}$ sites with $\mathrm{Yb}^{3+}$ neighbours to understand the observed features. Yet, our results somehow partially support Loh's hypothesis of some sort of $\mathrm{Yb}^{2+}-\mathrm{Yb}^{3+}$ pairs, in that some excited states have dual electronic structure with $\mathrm{Yb}^{2+}$ and $\mathrm{Yb}$-trapped exciton character at $\mathrm{Yb}-\mathrm{Cl}$ distances longer and shorter, respectively, than a critical value (note that the oxidation state of $\mathrm{Yb}$ in the Yb-trapped exciton is closer to 
III than to II; see Paper I). However, these type of states are not found below the $14 T_{1 \mathrm{u}}$ band, as commented above.

The maximum absolute error and root mean square deviation (rms) of the calculated energies relative to their experimental values for the whole absorption spectrum are 826 and $394 \mathrm{~cm}^{-1}$, respectively (see Table II).

\section{Excited state absorption spectrum.}

It has been recently suggested that excited-state absorption (ESA) may be used to probe the electronic and geometric structure of the $4 f^{N-1} 5 d$ excited states and the possibilities of ESA from the lowest excited state of $\mathrm{Yb}^{2+}$-doped $\mathrm{SrCl}_{2}$ have been considered in the framework of crystal field theory Hamiltonians. ${ }^{25}$

The electronic and geometric structure of $\mathrm{Yb}^{2+}$-doped $\mathrm{SrCl}_{2}$ excited states that could help to interpret ESA experiments have been presented in Table III of Paper I. They indicate that only two types of excitations could be expected: Intraconfigurational $1 E_{\mathrm{u}} \rightarrow i \Gamma_{u}$ excitations within the $4 f^{13}(7 / 2) 5 d\left(e_{g}\right)$ manifold and interconfigurational $4 f^{13}(7 / 2) 5 d\left(e_{g}\right)-1 E_{\mathrm{u}} \rightarrow i \Gamma_{u}$, where the $i \Gamma_{u}$ states have mixed $4 f^{13}(5 / 2) 5 d\left(e_{g}\right)$ and $4 f^{13}(7 / 2) 5 d\left(t_{2 g}\right)$ configurational character, according to the analyses of the spin-orbit wavefunctions in Table III of Paper I. The excitation energies from $1 E_{\mathrm{u}}$ would be below $5300 \mathrm{~cm}^{-1}$ and between 9500 and $17300 \mathrm{~cm}^{-1}$, respectively; the bandwidths would be very narrow and variable, respectively, following the values of $\mathrm{Yb}-\mathrm{Cl}$ distance offsets between the connected excited states (cf. Table III in Paper I). Excitation to the higher energy manifolds would have significant double-excitation character, which, together with the $u-u$ parity forbidden character of the whole ESA spectrum, would make their detection very unlikely (cf. Table III in Paper I).

\section{Emission spectrum of $\mathrm{SrCl}_{2}: \mathrm{Yb}^{2+}$.}

\section{Lowest emission bands.}

An early study of the emission spectrum of $\mathrm{Yb}^{2+}$-doped $\mathrm{SrCl}_{2}$ single crystals reported a very complex luminescence behavior when excited by near-ultraviolet radiation (334 and $313 \mathrm{~nm}$ mercury lines). ${ }^{6}$ Five emission bands were reported with band maxima in the 19000

- $26500 \mathrm{~cm}^{-1}$ spectral range and very complex variations of their relative intensities and 
band structure with temperature, excitation wavelength, and concentration. Two of these bands, with band maxima at 24700 and $26500 \mathrm{~cm}^{-1}$ at $4.2 \mathrm{~K}$ and long and short decay times, respectively, were assigned to vibronic emissions from $1 E_{\mathrm{u}}$ and $1 T_{2 \mathrm{u}}$, and to electric dipole allowed emission from $1 T_{1 \mathrm{u}}$ excited states to the $1 A_{1 \mathrm{~g}}$ ground state, respectively. Their electronic origins were investigated in absorption and emission spectra, as referred above, which suggests that the origins of the two bands are some $2000 \mathrm{~cm}^{-1}$ apart (see data of Refs. 5 and 6 in Table I). Our results also reveal the existence of these two bands; however, the energy separation between their origins is found to be higher than observed $\left(2800 \mathrm{~cm}^{-1}\right.$, see Table I) due to different theory-experiment discrepancies in the two origins.

The other three bands peaking at 19000, 23900, and $25400 \mathrm{~cm}^{-1}$ were ruled out as internal transitions of the $\mathrm{Yb}^{2+}$ ion and were called defect bands involving, possibly, $\mathrm{Yb}^{2+}-\mathrm{Yb}^{3+}$ pairs. ${ }^{6}$ The first two of them are significantly lower than the first energy level of $\mathrm{Yb}^{2+}$-doped $\mathrm{SrCl}_{2}$, therefore, they could only correspond to emissions connecting two excited states, if they were ascribable to $\mathrm{Yb}^{2+}$ impurities. This type of emissions requires that a very excited and metastable level is populated by either excited state absorption or by energy transfer between two excited impurities. ${ }^{26,27}$ However, the calculated energies and electronic structure of the excited manifolds of $\mathrm{Yb}^{2+}-\mathrm{SrCl}_{2}$, analyzed in detail in Paper I, indicate that such processes are unlikely when the excitation wavelengths are 334 and $313 \mathrm{~nm}$, given that the excited state absorptions and emissions would have a significant double-excitation character in addition of being $u-u$ parity forbidded transitions. Therefore, our calculations support the interpretation of them not being due to internal transitions of the $\mathrm{Yb}^{2+}$ ion.

The emission spectrum of $\mathrm{Yb}^{2+}$-doped $\mathrm{SrCl}_{2}$ under $355 \mathrm{~nm}$ excitation was measured again, much more recently, focusing on the variation of relative intensities and spontaneous emission lifetimes with temperature from 300 to $10 \mathrm{~K} .{ }^{10}$ This work revealed the presence of only two bands with band maxima separation of about $1800 \mathrm{~cm}^{-1}$ and lifetimes of the lower and higher energy bands of a few tenths of a millisecond and a microsecond, respectively. These results confirmed the observations and assignments of the two $\mathrm{Yb}^{2+} 1 E_{\mathrm{u}}, 1 T_{2 \mathrm{u}} \rightarrow 1 A_{1 \mathrm{~g}}$ and $1 T_{1 \mathrm{u}} \rightarrow 1 A_{1 \mathrm{~g}}$ bands of Ref. 6 , and identified the other three bands reported in Ref. 6 as due to impurities, since they were not observed. The emission lifetime of the higher energy band, measured at temperatures lower than $240 \mathrm{~K}$ (where the contribution of depopulation via nonradiative decay to $1 E_{\mathrm{u}}$ and $1 T_{2 \mathrm{u}}$ states should be negligible), has been reported to be in the $0.7-0.78 \mu \mathrm{s}$ range. The spontaneous emission lifetime calculated in this 
work using the energy $\left(30598 \mathrm{~cm}^{-1}\right)$ and electric dipole transition moment of $1 T_{1 \mathrm{u}}$ at its equilibrium distance ( $\mu^{2}=0.072498$ atomic units) is of the same order of magnitude than the experimental values: $0.247 \mu \mathrm{s}$ (it becomes $0.358 \mu \mathrm{s}$ when the calculated energy is shifted by $-3500 \mathrm{~cm}^{-1}$ ). The underestimation should be attributable mainly to the contributions from vibronic emissions from the close lying $2 T_{2 \mathrm{u}}$ and $1 A_{2 \mathrm{u}}$ levels, not included in the calculation.

As mentioned in Section II B 1, the presence of the low symmetry components of the $2 T_{2 \mathrm{u}}$ level was detected in the low energy side of the higher $1 T_{1 \mathrm{u}}$ band $\left(7 \mathrm{~cm}^{-1}\right.$ below $1 T_{1 \mathrm{u}}$; see Table I). However these signals are observed only at very low temperatures (2 and $4.2 \mathrm{~K}$ ) and under uniaxial compression or external fields, as referred above. ${ }^{8,9}$ The calculations place this level below $1 T_{1 \mathrm{u}}$ as well, but at larger energy separation: $266 \mathrm{~cm}^{-1}$. In addition, the calculations find the $1 A_{2 \mathrm{u}}$ level lying close to both excited states (see Table I), however, none of the experimental signals observed below $1 T_{1 \mathrm{u}}$ have been attributed to its vibronic transitions, as far as we know.

\section{Emissions from higher energy levels.}

As we have just discussed, the two emission bands experimentally observed after nearultraviolet excitation (335 to $313 \mathrm{~nm} ; 29800-31900 \mathrm{~cm}^{-1}$ ) are due to excited states of the $4 f^{13}(7 / 2) 5 d\left(e_{g}\right)$ manifold: $1 E_{\mathrm{u}}$ and $1 T_{2 \mathrm{u}}$ (lower energy band), and $1 T_{1 \mathrm{u}}$ (higher energy band). We examine now whether emission bands from higher energy levels can be predicted for the $\mathrm{SrCl}_{2}: \mathrm{Yb}^{2+}$, so that they could be investigated experimentally by exciting the sample above $31900 \mathrm{~cm}^{-1}$ and below the host absorption $\left(7.50 \mathrm{eV} ; 60000 \mathrm{~cm}^{-1}\right)^{28}$.

To do this, we have analyzed the stability of higher excited states versus nonradiative decay, in particular, multiphonon relaxation to lower lying levels, and we have collected the relevant data of the potentially emitting levels in Table IV. The results suggest that three additional emission bands could be observed with origins at 33800, 36400, and $43600 \mathrm{~cm}^{-1}$ due to the superposition of emissions from $4 E_{\mathrm{u}}$ and $5 T_{2 \mathrm{u}} ; 6 T_{2 \mathrm{u}}, 5 E_{\mathrm{u}}$, and $6 T_{1 \mathrm{u}} ;$ and $5 A_{1 \mathrm{u}}$. To support this conclusion, we have estimated the approximate order, $p$, of the multiphonon process that could compete with the radiative emission from each level $\mathrm{i} \Gamma$, by depopulating it as proposed in Ref. 29: $p=E_{\text {gap }}(\mathrm{i} \Gamma) / \hbar \nu_{\max }$ (see Table IV). For this, we have used the energy gap below state $i \Gamma, E_{\text {gap }}(\mathrm{i} \Gamma)$, and the $\mathrm{YbCl}_{8}$ moiety breathing mode vibrational fre-

quency value of the level lying below the gap as maximum phonon frequency, $\nu_{\max }$ (all the 
data needed have been reported in Paper I). The values of $p$ make it reasonable to expect that multiphonon relaxation could be less efficient in depopulating the $4 E_{\mathrm{u}}$ excited state, whereas it should compete with radiative decay in the case of $6 T_{2 \mathrm{u}}$ and $5 A_{1 \mathrm{u}}$. As a reference, the calculated multiphonon order of $1 T_{1 \mathrm{u}}$ is $p=12$ and it has been shown that this level retains enough population to be emissive even at room temperature and that its emission relative intensity increases as temperature is diminished, following the expected decrease of multiphonon rate (and depopulation) with decreasing temperature. The different spin character of the states lying above and below the gaps also supports the stability of the former. So, whereas $4 E_{\mathrm{u}}$ and $5 A_{1 \mathrm{u}}$ are high-spin states (having more than $80 \%$ contribution from a single $S=1$ term), the states lying below them are mixed-spin states (showing extensive mixing of $S=0$ and 1 terms), the contrary is true for $6 T_{2 \mathrm{u}}$ and the state lying below it. Finally, energy barriers for crossings to lower excited states of about $1000 \mathrm{~cm}^{-1}$ have been found above $6 T_{2 \mathrm{u}}$ and $5 A_{1 \mathrm{u}}$ which could quench their emissions if excited above the respective energy barriers. This characteristic and the competitive multiphonon depopulation commented above suggest that the emissions from $6 T_{2 \mathrm{u}}$ and $5 A_{1 \mathrm{u}}$ are more likely to be observed if the excitation energies chosen are close to the predicted origins and the experiments are done at very low temperatures.

\section{CONCLUSIONS}

We have calculated the absorption and emission spectra of $\mathrm{Yb}^{2+}$-doped $\mathrm{SrCl}_{2}$ crystals using $a b$ initio methods of quantum chemistry and have reassigned and reinterpreted the available experimental data. The root mean square deviations between calculated and experimental electric dipole allowed transitions in the absorption spectrum is found to be $394 \mathrm{~cm}^{-1}$ once all the calculated energies are shifted by $-3500 \mathrm{~cm}^{-1}$ to account for a systematic overestimation of the energy of all excited states with respect to the ground state, due to an overcorrection of the correlation energy of the latter by the correlation methods used. The bands in the absorption spectra appear to be grouped in three regions separated by energy gaps. The excited states of the first region $\left(27000-29300 \mathrm{~cm}^{-1}\right)$ have a dominant $4 f^{13}(7 / 2) 5 d\left(e_{g}\right)$ configurational character; their $\mathrm{YbCl}_{8}$ moiety breathing mode vibrational frequencies show very good agreement with the experimental values and the sign and magnitude of their $\mathrm{Yb}-\mathrm{Cl}$ bond length offshifts with respect to the ground 
state bond length are consistent with spectroscopic results at low temperatures under uniaxial compression. The states of the second region $\left(34500-41500 \mathrm{~cm}^{-1}\right)$ show strong $4 f^{13}(5 / 2) 5 d\left(e_{g}\right)$ and $4 f^{13}(7 / 2) 5 d\left(t_{2 g}\right)$ configurational mixing, which results in vibrational progressions dominated by the zero-phonon line, indicating that the 6 unstructured experimental band maxima observed in this part of the spectrum at very low temperature, are electronic origins. The first band of the third region is one of the most intense and broad bands in the whole absorption spectrum. It reflects the complex electronic structure characteristic of this part of the spectrum $\left(44700-50500 \mathrm{~cm}^{-1}\right)$, where the excited states show spin-orbit induced mixing between the double-well $\left[4 f^{13}(7 / 2) a_{1 g}^{Y b T E}\right.$ Yb-trapped exciton $4 f^{13}(7 / 2) 6 s$ impurity-state] and $4 f^{13}(5 / 2) 5 d\left(t_{2 g}\right)$ configurational characters; in particular, it gets its intensity from its $4 f^{13}(5 / 2) 5 d\left(t_{2 g}\right)$ configurational character (more precisely, from a significant $5^{1} T_{1 \mathrm{u}}$ spin-enabling contribution) and its width from its $4 f^{13}(7 / 2) 6 s$ character, which is also responsible for a very long bond length offshift. The accuracy of the calculated emission bands is comparable to that of the absorption spectrum, and the experimental and calculated emission lifetime of the only observed electric dipole allowed emission compare as follows: $\tau_{\text {exp }}=0.7-0.78 \mu s ; \tau_{\text {theo }}=0.247-0.358 \mu \mathrm{s}$.

Some predictions emerge from the $a b$ initio calculations which suggest that more experimental work should be done in this material. First, photoionization should occur above $49000 \mathrm{~cm}^{-1}$; this prediction could be proved experimentally by monitoring the appearence of characteristic $\mathrm{Yb}^{3+}$-doped near-infrared emission upon photoionization. Second, three new emission bands should be observable above the two known emission bands of $\mathrm{Yb}^{2+}$ doped $\mathrm{SrCl}_{2}$; their origins should be at about $33800 \mathrm{~cm}^{-1}$ (slow emission), $36400 \mathrm{~cm}^{-1}$ $(\tau \sim 4.2 \mu \mathrm{s})$, and $43600 \mathrm{~cm}^{-1}$ (slow emission). For the last two bands, multiphonon decay should be competitive, so, their relative intensities should increase at low temperatures; also, the excitation energies chosen should be close to the predicted origins since energy barriers for crossings to lower excited states, of about $1000 \mathrm{~cm}^{-1}$, have been found in their potential energy surfaces.

\section{Acknowledgments}

This research was supported in part by the Ministerio de Ciencia e Innovación, Spain, under contract MAT2008-05379. G.S-S. acknowledges an FPI fellowship from the Ministerio 
de Ciencia e Innovación, Spain.

* Corresponding author; Electronic address: zoila.barandiaran@uam.es

1 T. Justel, H. Nikol, and C. Ronda, Angew. Chem. Int. Ed. 37, 3085 (1998).

2 S. Kück, Appl. Phys. B 72, 515 (2001).

3 J. Y. Tsao, IEEE Circuits \& Devices 20, 28 (2004).

4 D. S. McClure and Z. Kiss, J. Chem. Phys. 39, 3251 (1963).

5 T. S. Piper, J. P. Brown, and D. S. McClure, J. Chem. Phys. 46, 1353 (1967).

6 H. Witzke, D. S. McClure, and B. Mitchell, in Luminescence of Crystals, Molecules, and Solutions, edited by F. E. Williams (Plenum, New York, 1973), p. 598.

7 E. Loh, Phys. Rev. B 7, 1846 (1973).

8 A. A. Kaplyanskii and P. L. Smolyanskii, Sov. Phys. Solid State 17, 357 (1975).

9 A. A. Kaplyanskii and P. L. Smolyanskii, Opt. Spectrosc. 40, 301 (1976).

10 Z. Pan, C. Duan, and P. A. Tanner, Phys. Rev. B 77, 085114 (2008).

11 G. Sánchez-Sanz, L. Seijo, and Z. Barandiarán, J. Chem. Phys. 131, 024505 (2009).

12 J. Rubio, J. Phys. Chem. Solids 52, 101 (1991).

13 G. Sánchez-Sanz, L. Seijo, and Z. Barandiarán, J. Chem. Phys., in press.

14 B. Ordejón, V. Vallet, J.-P. Flament, L. Seijo, and Z. Barandiarán, J. Lumin. 126, 779 (2007).

15 V. Vallet, L. Maron, C. Teichteil, and J.-P. Flament, J. Chem. Phys. 113, 1391 (2000).

16 F. Ruipérez, L. Seijo, and Z. Barandiarán, J. Chem. Phys. 122, 234507 (2005).

17 R. Valiente, F. Rodríguez, J. González, H. U. Güdel”, R. Martín-Rodríguez, L. Nataf, M. N. Sanz-Ortiz and K. Krämer, Chem. Phys. Lett. 481, 149 (2009).

18 C. S. Yoo, H. B. Radousky, N. C. Holmes and N. M. Edelstein, Phys. Rev. B 44, 830 (1991).

19 J. L. Pascual, Z. Barandiarán, and L. Seijo, Phys. Rev. B 76, 104109 (2007).

20 E. J. Heller, J. Chem. Phys. 62, 1544 (1975).

21 E. J. Heller, Acc. Chem. Res. 14, 368 (1981).

22 J. I. Zink and K. S. Shin, Molecular distortions in excited electronic states determined from electronic and resonance raman spectroscopy (Wiley, New York, 1991), vol. 16 of Advances in Photochemistry, pp. 119-214.

23 G. Sánchez-Sanz, L. Seijo, and Z. Barandiarán, J. Phys. Chem. A 113, 12591 (2009). 
24 G. Sánchez-Sanz, L. Seijo, and Z. Barandiarán, Spec. Letters 43, 393 (2010).

25 M. F. Reid, L. Hu, S. Frank, C. Duan, S. Xia and M. Yin, Eur. J. Inorg. Chem. 2010, 2649 (2010).

26 J. Grimm and H. U. Güdel, Chem. Phys. Lett. 404, 40 (2005).

27 E. Beurer, J. Grimm, P. Gerner and H. U. Güdel, J. Amer. Chem. Soc. 128, 3110 (2006).

28 C. Sugiura, Phys. Rev. B 9, 2679 (1974).

29 L. A. Riseberg and H. W. Moos, Phys. Rev. 174, 429 (1968). 
TABLE I: Energy levels of the $4 f^{14}$ and $4 f^{13}(7 / 2) 5 d\left(e_{g}\right)$ configurations for $\mathrm{SrCl}_{2}$ : $\mathrm{Yb}^{2+}$. Theoretical and experimental results. $\mathrm{Yb}-\mathrm{Cl}$ bond distances, $R_{e}$, in $\AA$; totally symmetric vibrational frequencies, $\bar{\nu}_{a_{1 g}}$, and zero-phonon energies, in $\mathrm{cm}^{-1}$. Values not observed directly are given in square parentheses.

\begin{tabular}{|c|c|c|c|c|c|c|c|c|c|c|}
\hline \multirow[t]{3}{*}{ State } & \multirow{3}{*}{$\begin{array}{c}R_{e} \\
\text { theory }\end{array}$} & \multicolumn{5}{|c|}{ zero-phonon line } & \multicolumn{4}{|c|}{$\bar{\nu}_{a_{1 g}}$} \\
\hline & & theory & (error) & \multicolumn{3}{|c|}{ experiment } & \multirow[t]{2}{*}{ theory } & \multicolumn{3}{|c|}{ experiment } \\
\hline & & & & Refs. 5,6 & Ref. 9 & Ref. 10 & & Ref. 5 & Ref. 9 & Ref. 10 \\
\hline $1 A_{1 \mathrm{~g}}$ & 2.955 & 0 & & 0 & 0 & 0 & 189 & & $206 \pm 2^{a}$ & $209 \pm 2^{b}$ \\
\hline $1 E_{\mathrm{u}}$ & 2.906 & 27781 & $(2800)$ & {$[24958-25088]^{c}$} & & {$[25252]^{d}$} & 197 & & & \\
\hline $1 T_{2 \mathrm{u}}$ & 2.906 & 27828 & & & & & 196 & & & \\
\hline $2 T_{2 \mathrm{u}}$ & 2.898 & 30332 & $(3294)$ & & {$[27038]^{e}$} & & 208 & & & \\
\hline $1 A_{2 \mathrm{u}}$ & 2.897 & 30357 & & & & & 210 & & & \\
\hline $1 T_{1 \mathrm{u}}$ & 2.900 & 30598 & $(3549)$ & $27049^{f}$ & $27045^{a, f}$ & $27049^{g}$ & 203 & $215^{f}$ & $210 \pm 3^{f}$ & $213 \pm 3^{g}$ \\
\hline $2 E_{\mathrm{u}}$ & 2.900 & 31125 & & & & & 203 & & & \\
\hline $1 A_{1 \mathrm{u}}$ & 2.899 & 31151 & & & & & 206 & & & \\
\hline $2 T_{1 \mathrm{u}}$ & 2.899 & 31269 & & & & & 205 & & & \\
\hline $3 T_{2 \mathrm{u}}$ & 2.900 & 31538 & & & & & 203 & & & \\
\hline $3 T_{1 \mathrm{u}}$ & 2.900 & 31606 & $(3161)$ & & & $(28377)^{g}$ & 200 & & & $213 \pm 3^{g}$ \\
\hline $3 E_{\mathrm{u}}$ & 2.898 & 32590 & & & & & 207 & & & \\
\hline $4 T_{1 \mathrm{u}}$ & 2.896 & 32795 & $(3746)$ & & & $29049^{g}$ & 207 & $215^{f}$ & & \\
\hline $4 T_{2 \mathrm{u}}$ & 2.894 & 33058 & & & & & 209 & & & \\
\hline
\end{tabular}

\footnotetext{
${ }^{a}$ From vibrational progression in emission spectra at $4.2 \mathrm{~K}$.

${ }^{b}$ From vibrational progression in emission spectra at $10 \mathrm{~K}$.

${ }^{c}$ Electronic origin should lie between these values. See text for details.

${ }^{d}$ From extrapolation of the $1 T_{1 \mathrm{u}}-1 E_{\mathrm{u}}$ maxima separation from the room temperature emission spectrum to $10 \mathrm{~K}$.

${ }^{e}$ Not observed directly; value deduced from effects of applied fields on emission spectra at 4.2 and $1.8 \mathrm{~K}$. ${ }^{f}$ From vibrational progression in absorption spectra at $4.2 \mathrm{~K}$.

${ }^{g}$ From vibrational progression in absorption spectra at $10 \mathrm{~K}$. In parentheses: broad structure centered at $28590 \mathrm{~cm}^{-1}$, tentatively assigned to the first member of the $213 \mathrm{~cm}^{-1}$ progression of $3 T_{1 \mathrm{u}}$.
} 
TABLE II: Theoretical and experimental relative energies and absorption oscillator strengths of $\mathrm{i} T_{1 \mathrm{u}}$ levels with respect to $1 T_{1 \mathrm{u}}$ for $\mathrm{Yb}^{2+}$-doped $\mathrm{SrCl}_{2}$. Calculated bond length offsets with respect to ground state, $\Delta R_{e}\left(\mathrm{i} T_{1 \mathrm{u}}\right)=R_{e}\left(\mathrm{i} T_{1 \mathrm{u}}\right)-R_{e}\left(1 A_{1 \mathrm{~g}}\right)$, are given in $\AA$. Experimental energies are referred to the $1 T_{1 \mathrm{u}}$ zero-phonon energy $27049 \mathrm{~cm}^{-1}$ from the absorption spectrum of Ref. 5 and are listed according to this work's assignment; additional data and special assignments from Refs. 10 and 7 are also given. Errors of theoretical energies relative to experimental values of Ref. 5, are given in parentheses; their root mean square deviation, rms, is also given. Energies are given in $\mathrm{cm}^{-1}$. See text for details.

theoretical $^{1}$

experimental

$\begin{array}{lll}\text { Ref. } 5^{2} & \text { Ref. } 10^{3} & \text { Ref. } 7\end{array}$

level $\Delta R_{e} \quad$ energy (error) $f_{i} / f_{1} \quad$ energy $f_{i} / f_{1}$ energy $f_{i} / f_{1}$ assignment energy assignment $^{4}$

Region $1,4 f^{13}(7 / 2) 5 d\left(e_{g}\right)$ levels

$\begin{array}{ccccccccccc}1 & T_{1 u} & -0.055 & 0 & 0 & 1.000 & 0 & 1.0 & 0 & 0 & 1 \\ 2 & T_{1 u} & -0.056 & 671 & & 0.059 & & & & & \\ 3 & T_{1 u} & -0.055 & 1008 & -320 & 0.065 & & & (1328) & 1451 & 1^{\prime} \\ 4 & T_{1 u} & -0.059 & 2197 & 187 & 0.002 & 2010 & 0.04 & 2000 & & 1^{\prime \prime}\end{array}$

Region $2,4 f^{13}(5 / 2) 5 d\left(e_{g}\right)+4 f^{13}(7 / 2) 5 d\left(t_{2 g}\right)$ levels

\begin{tabular}{|c|c|c|c|c|c|c|c|c|c|c|}
\hline $5 T_{1 u}$ & -0.011 & 7505 & -484 & 0.553 & 7989 & 0.15 & 0.13 & $7 / 2, \mathrm{~s}$ & & II-III $7 / 2, e_{g}$ \\
\hline $6 T_{1 u}$ & -0.012 & 9507 & & 0.042 & & & & & 9051 & II-III \\
\hline $7 T_{1 u}$ & -0.011 & 9942 & -228 & 0.153 & 10170 & 0.07 & 0.16 & & & II-III \\
\hline $8 T_{1 u}$ & -0.007 & 10544 & -32 & 0.619 & 10576 & 0.1 & 0.20 & & & $15 / 2, e_{g}$ \\
\hline $9 T_{1 u}$ & +0.006 & 11938 & & 0.002 & & & & & & \\
\hline $10 T_{1 u}$ & -0.004 & 12171 & 472 & 1.246 & 11699 & 1.9 & 1.1 & & & $1^{\prime}$ \\
\hline $11 T_{1 u}$ & -0.024 & 12565 & & 1.629 & & & & & & \\
\hline $12 T_{1 u}$ & -0.013 & 12909 & 209 & 0.361 & 12700 & 0.59 & 1.1 & & & $1^{\prime \prime}$ \\
\hline \multirow[t]{3}{*}{$13 T_{1 u}$} & -0.010 & 14478 & 438 & 7.935 & 14040 & 3.4 & 2.4 & & & $17 / 2, t_{2 g}$ \\
\hline & & & & & & & & & 15151 & $1^{\prime}$ \\
\hline & & & & & & & & & 15701 & $1^{\prime \prime}$ \\
\hline
\end{tabular}


Region $3,4 f^{13}(5 / 2) 5 d\left(t_{2 g}\right)+\left[4 f^{13}(7 / 2) a_{1 g}^{Y b T E}-4 f^{13}(7 / 2) 6 s\right]$ levels

\begin{tabular}{|c|c|c|c|c|c|c|c|c|c|}
\hline $14 T_{1 u}+0.116$ & 17711 & $-362^{5}$ & 8.381 & 18745 & 1.1 & 0.2 & $5 / 2, \mathrm{~s}$ & & II-III $5 / 2, e_{g}$ \\
\hline $15 T_{1 u}+0.121$ & 18203 & & 0.001 & & & & & & \\
\hline \multirow[t]{2}{*}{$16 T_{1 u}+0.056$} & 19307 & -80 & 0.644 & 19387 & 1.1 & 0.4 & & & II-III \\
\hline & & & & & & & & 20851 & II-III \\
\hline $17 T_{1 u}+0.020$ & 20700 & -826 & 14.856 & 21526 & 4.4 & 2.8 & & & II-III $7 / 2, t_{2 g}$ \\
\hline $18 T_{1 u}+0.013$ & 21256 & & 0.045 & & & & & 22651 & II-III \\
\hline $19 T_{1 u}+0.012$ & 22914 & -440 & 0.113 & 23354 & 1.2 & 1.8 & & & II-III \\
\hline $20 T_{1 u}+0.010$ & 23466 & & 0.147 & & & & & & \\
\hline $21 T_{1 u}+0.125$ & 28397 & & 0.009 & & & & & & \\
\hline $\mathrm{rms}$ & & 394 & & & & & & & \\
\hline
\end{tabular}

1 Energies correspond to adiabatic differences: $T_{e}\left(\mathrm{i} T_{1 \mathrm{u}}\right)-T_{e}\left(1 T_{1 \mathrm{u}}\right)$. Relative absorption oscillator strengths, $f_{i} / f_{1}$, are calculated at the ground state $\mathrm{Yb}-\mathrm{Cl}$ equilibrium distance $2.955 \AA$.

2 From absorption spectrum at $4.2 \mathrm{~K}$. Energies correspond to $\mathrm{i} T_{1 \mathrm{u}}$ band maxima and are referred to $1 T_{1 \mathrm{u}}$ zero-phonon line at $27049 \mathrm{~cm}^{-1}$, except for the $4 T_{1 \mathrm{u}}\left(29272 \mathrm{~cm}^{-1}\right)$ and $16 T_{1 \mathrm{u}}\left(46649 \mathrm{~cm}^{-1}\right)$ band maxima, which are referred to the $1 T_{1 \mathrm{u}}$ band maximum at $27262 \mathrm{~cm}^{-1}$ (Ref. 5). See text for details.

3 From absorption spectrum at $10 \mathrm{~K}$. Tentative assignment in parentheses. Energies correspond to $\mathrm{i} T_{1 \mathrm{u}}$ - $1 T_{1 \mathrm{u}}$ zero-phonon line energy differences. Experimental relative oscillator strengths from Ref. 5 with background corrections from Ref. 10 .

$41,1^{\prime}, 1^{\prime \prime}$ : three types of isolated $\mathrm{Yb}^{2+}$. II-III: three types of $\mathrm{Yb}^{2+}$ sites with $\mathrm{Yb}^{3+}$ neighbors.

${ }^{5}$ Error of the calculated $14 T_{1 \mathrm{u}}$ band peak at $18383 \mathrm{~cm}^{-1}$. 
TABLE III: Analysis of the $13 T_{1 \mathrm{u}}, 14 T_{1 \mathrm{u}}$, and $17 T_{1 \mathrm{u}}$ spin-orbit wave functions at different $\mathrm{Yb}-\mathrm{Cl}$ distances. Only the weights of spin-orbit free wave functions larger than $5 \%$ are given.

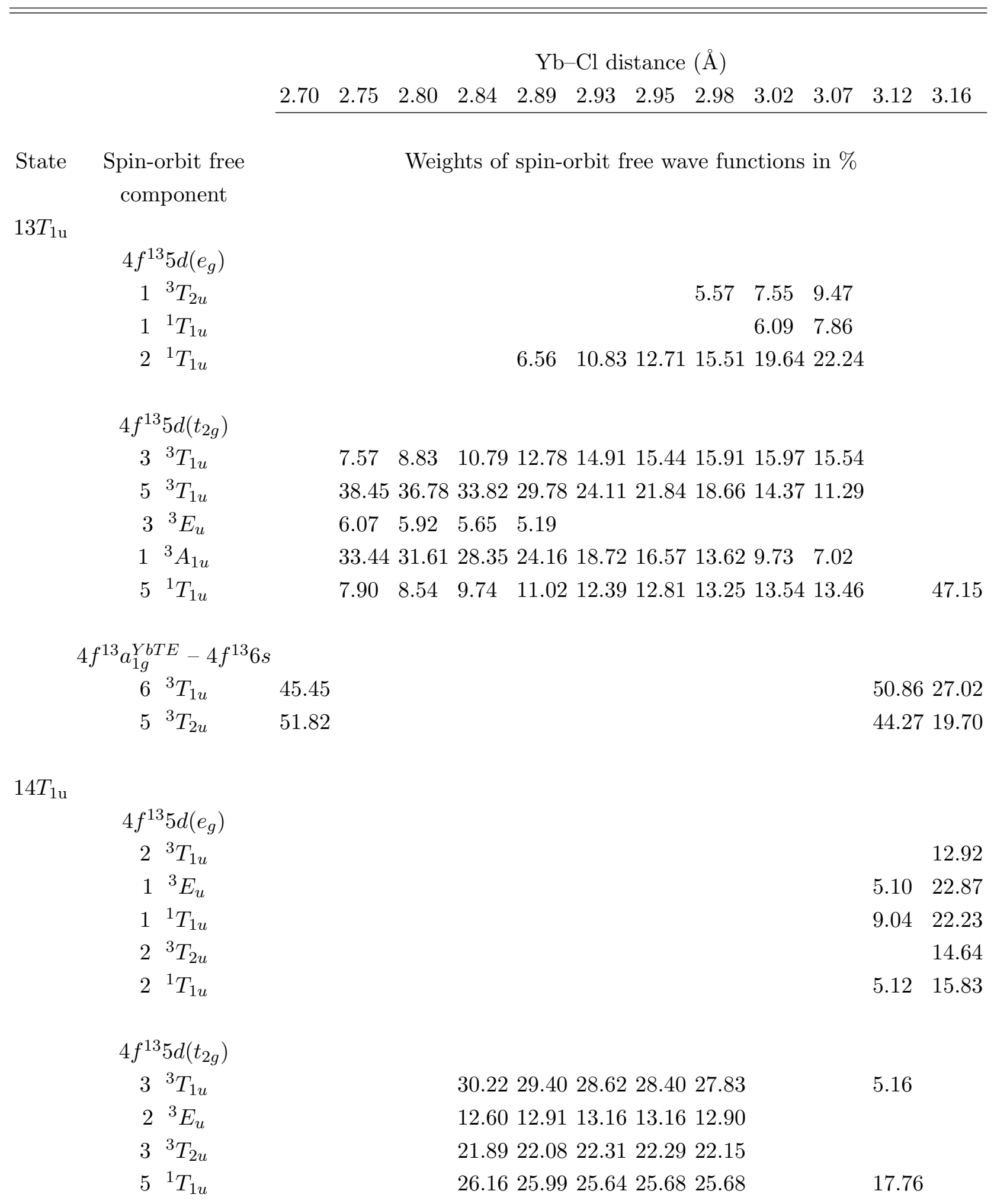




$$
\begin{aligned}
& 4 f^{13} a_{1 g}^{Y b T E}-4 f^{13} 6 s \\
& { }^{6}{ }^{3} T_{1 u} \quad 34.0436 .1936 .64 \\
& { }^{5}{ }^{3} T_{2 u} \quad 14.7157 .3057 .12
\end{aligned}
$$

\begin{tabular}{|c|c|c|c|c|c|c|c|c|}
\hline \multicolumn{9}{|c|}{$4 f^{13} 5 d\left(t_{2 g}\right)$} \\
\hline $4^{3} T_{1 u}$ & \multicolumn{8}{|c|}{5.13} \\
\hline $2^{3} E_{u}$ & 19.06 & 16.6 & 15.97 & & & & $12.9614 .82 \quad 15.0314 .63$ & 15.2614 .73 \\
\hline $3^{3} T_{2 u}$ & 25.43 & 21.21 & 20.85 & & & 8.13 & $20.82 \quad 20.32 \quad 20.3820 .28$ & 15.9318 .90 \\
\hline $3^{1} T_{1 u}$ & & & & & & 13.16 & & \\
\hline $4^{3} T_{2 u}$ & & & & & 18.49 & & & \\
\hline $5^{3} T_{1 u}$ & & & & & 37.14 & & & \\
\hline $3^{3} E_{u}$ & & & & & 12.80 & & & \\
\hline $4{ }^{1} T_{1 u}$ & 6.52 & 5.81 & 5.59 & & & 5.09 & 5.21 & \\
\hline $1^{3} A_{1 u}$ & & & & & 17.48 & & & \\
\hline $5^{1} T_{1 u}$ & 34.71 & 41.6 & 43.03 & & & & 43.5845 .3045 .2045 .71 & \\
\hline \multicolumn{9}{|c|}{$4 f^{13} a_{1 g}^{Y b T E}-4 f^{13} 6 s$} \\
\hline $6^{3} T_{1 u}$ & & & & 40.15 & & 18.35 & & \\
\hline $5{ }^{3} T_{2 u}$ & & & & & & 10.30 & & \\
\hline $6^{1} T_{1 u}$ & & & & 39.47 & & 28.75 & & 44.7447 .32 \\
\hline
\end{tabular}

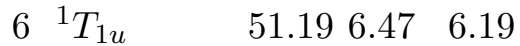

$17 T_{1 \mathrm{u}}$
51.7050 .7518 .03

$47.20 \quad 47.90 \quad 7.21$

16.78 
TABLE IV: Data of potentially emitting levels under high energy excitation and characteristics of the predicted emission bands. Calculated origin of emission bands are shifted $-3500 \mathrm{~cm}^{-1}$. All energies are given in $\mathrm{cm}^{-1}$. See text for details.

\begin{tabular}{ccccc}
\hline \hline levels & $\begin{array}{c}\text { energy gap } \\
\text { below level }\end{array}$ & $p^{a}$ & $\begin{array}{c}\text { emission } \\
\text { lifetime }\end{array}$ & $\begin{array}{c}\text { origin of } \\
\text { emission band }\end{array}$ \\
$4 E_{\mathrm{u}}, 5 T_{2 \mathrm{u}}$ & 4200 & 20 & slow & 33800 \\
$6 T_{2 \mathrm{u}}, 5 E_{\mathrm{u}}$, & 1700 & 9 & slow & 36400 \\
$6 T_{1 \mathrm{u}}$ & & & $4.200 \mu s$ & $36600^{b}$ \\
$5 A_{1 \mathrm{u}}$ & 2000 & slow & $43600^{b}$ \\
& & & \\
\hline \hline
\end{tabular}

${ }^{a}$ Multiphonon order, $p=E_{g a p}(i \Gamma) / \hbar \nu_{\max }$. See text for details.

${ }^{b}$ Excitation should be done below the barrier for crossing to lower states; the barrier height is $\sim 1000 \mathrm{~cm}^{-1}$ above the electronic origin. 
FIG. 1: $\mathrm{YbCl}_{8}$ moiety breathing mode potential energy curves of the states of the $\left(\mathrm{YbCl}_{8} \mathrm{Sr}_{12}\right)^{18+}$ cluster embedded in $\mathrm{SrCl}_{2}$ with dominant configurational character $4 f^{13} 5 d\left(e_{g}\right)$ (red), $4 f^{13} 5 d\left(t_{2 g}\right)$ (blue), and $\left[4 f^{13} a_{1 g}^{Y b T E}-4 f^{13} 6 s\right]$ (green), in increasing energy order. All results include $\mathrm{SrCl}_{2}$ host embedding, scalar relativistic effects, and 96 valence electron correlation. Left: spin-orbit free results. Right: spin-orbit results. States with strong mixing of configurational character are shown in black.

FIG. 2: Lowest bands in absorption spectra of $\mathrm{Yb}^{2+}$-doped $\mathrm{SrCl}_{2}$. a (upper horizontal scale): Experimental spectrum at $4.2 \mathrm{~K}$ of Ref. 9. b (lower horizontal scale): Vibrational progressions on the $a_{1 g}$ vibrational mode for the lowest three $T_{1 \mathrm{u}}$ states (see Table I). Inset: Vibrational progression of the $1 T_{1 \mathrm{u}}$ state calculated using $\left|R_{e}\left(1 T_{1 \mathrm{u}}\right)-R_{e}\left(1 A_{1 \mathrm{~g}}\right)\right|=0.075 \AA$. See text for details.

FIG. 3: Calculated absorption spectrum of $\mathrm{Yb}^{2+}$-doped $\mathrm{SrCl}_{2}$ using spin-orbit CI data from Table II and different values for the line broadening parameter $\left(35\right.$ and $5 \mathrm{~cm}^{-1}$ in the low and high resolution spectra, respectively); the higher energy scale shows the calculated energies shifted by $-3500 \mathrm{~cm}^{-1}$. The most intense $T_{1 \mathrm{u}}$ electronic origins are indicated with the ordinals used in Table II. Some electric dipole forbidden origins are indicated with vertical bars. The potential energy curves of all states of $T_{1 \mathrm{u}}$ symmetry are plotted in the inset; their energies are referred to that of $1 T_{1 \mathrm{u}}$; their spectroscopic constants are given in Table III of Paper I. States 5,14 , and $21 T_{1 \mathrm{u}}$ are indicated and the energy curves of 13,14 , and $17 T_{1 \mathrm{u}}$ are plotted with thicker lines to facilitate visualization. The $\mathrm{Yb}-\mathrm{Cl}$ equilibrium geometry of the ground state is indicated with a vertical dotted line at $2.955 \AA$. 
without spin-orbit

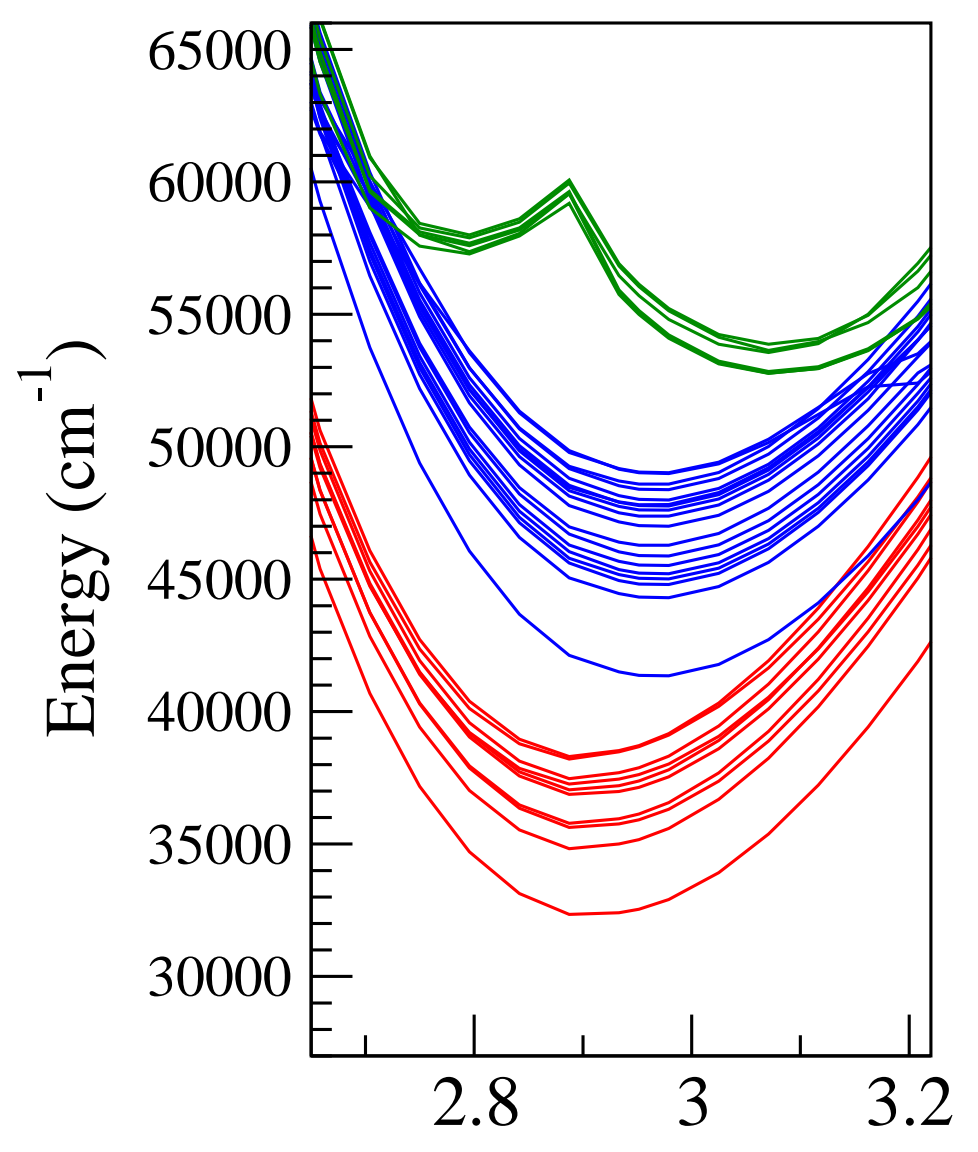

with spin-orbit

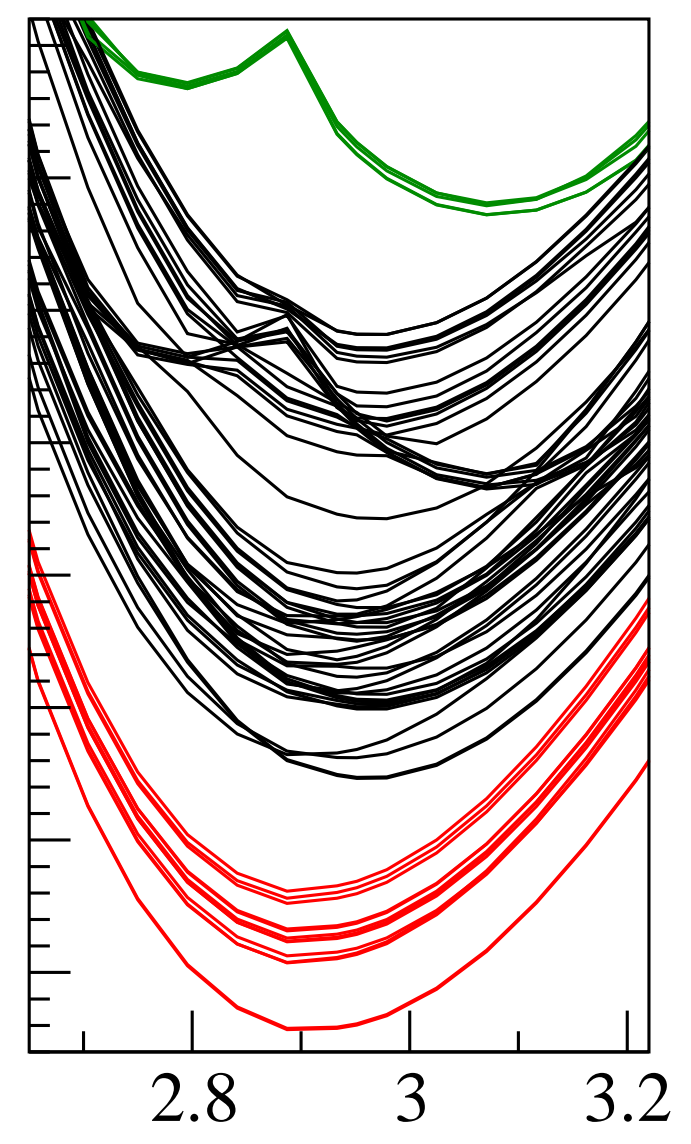

$\mathrm{R}(\mathrm{Yb}-\mathrm{Cl}) / \AA$ 


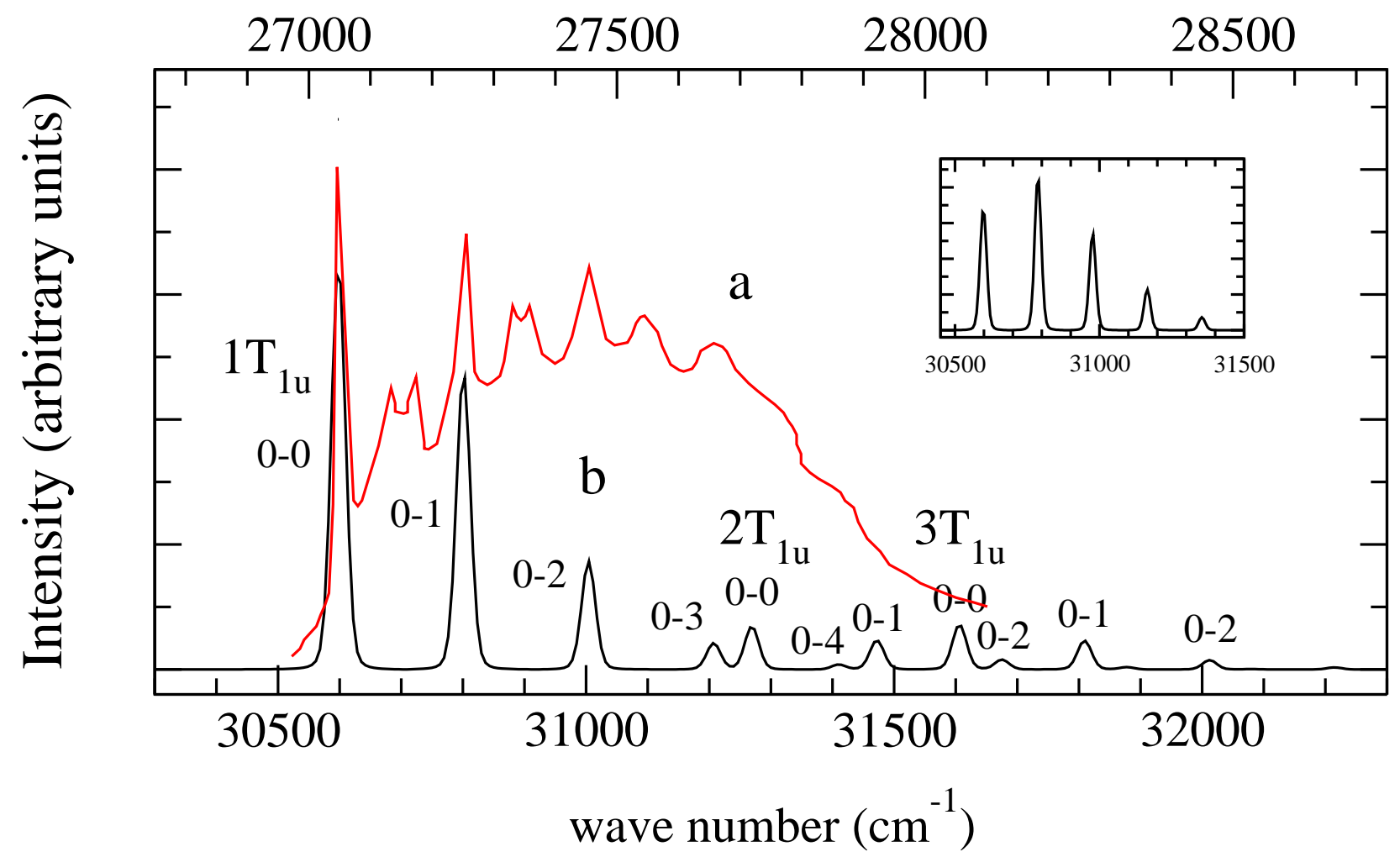




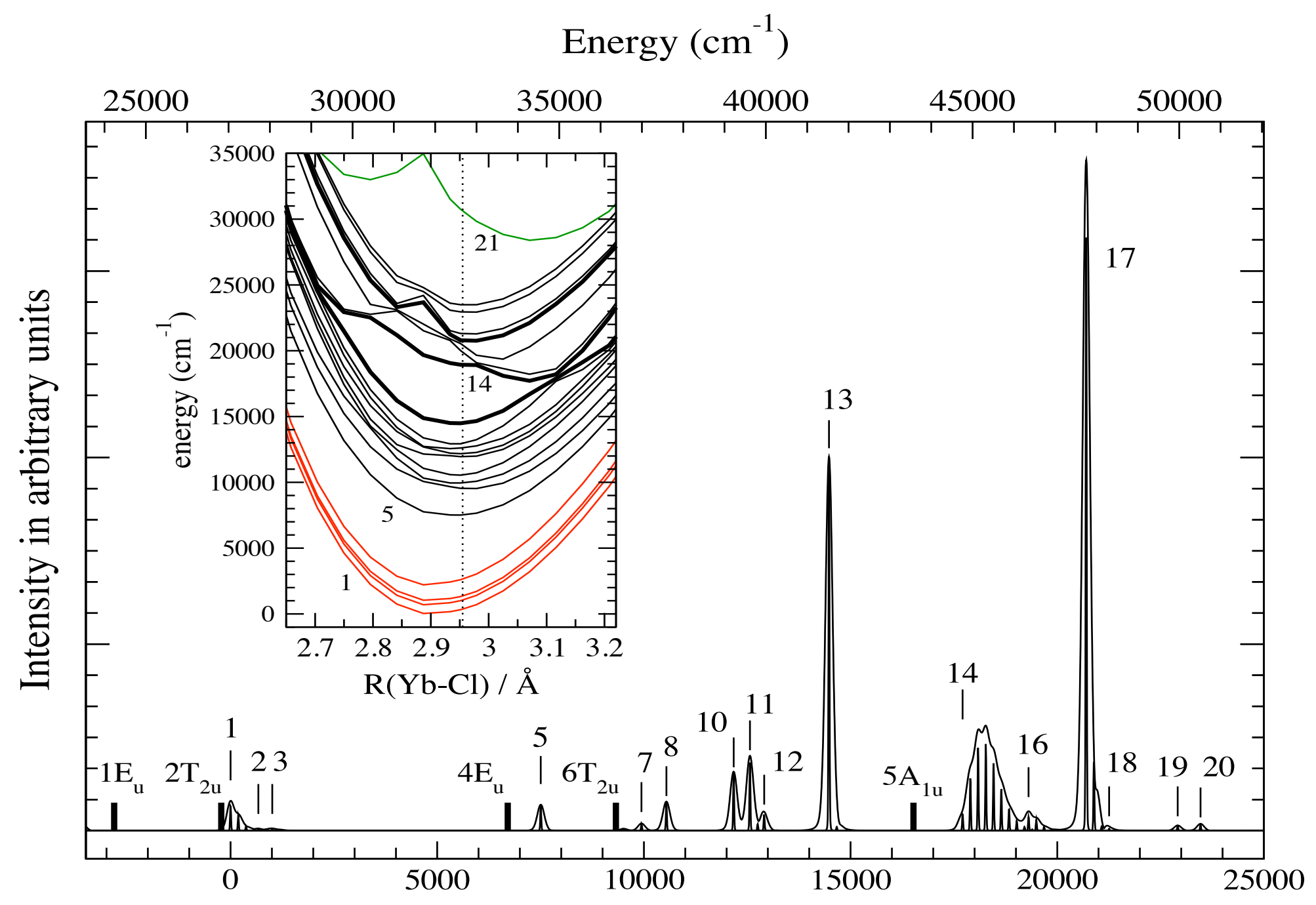

Energy relative to $1 \mathrm{~T}_{1 \mathrm{u}}$ zero-phonon line $/ \mathrm{cm}^{-1}$ 